\title{
Inborn Errors of Metabolism in Pediatric Epilepsy
}

Anna S. Cosnahan, PharmD and Christopher T. Campbell, PharmD

Patients with refractory epilepsy from inborn errors of metabolism typically present as neonates. Direct supplementation with the deficient vitamin or cofactor is recommended, and case series report both efficacy and safety data of these agents. Some conditions may also occur together, necessitating multiple treatments. Despite effective and early treatment, patients are at heightened risk for neurological sequela. The literature on seizures related to metabolic deficiencies for pediatric patients is limited but has some guidance on appropriate dosing and monitoring for agents to target specific deficiencies, which may help with narrowing antiepileptic therapies, reducing side effects, and improving neurodevelopmental outcomes and quality of life. The focus of this review is to discuss the pharmacotherapy, including the most updated published efficacy and safety data, involved in treating refractory epilepsy as a result of metabolic errors.

ABBREVIATIONS AAA, alpha-aminoadipic acid; AASA, alpha-aminoadipic semialdehyde; ALDH7A1, aldehyde dehydrogenase (antiquitin); CSF, cerebrospinal fluid; FARS, folinic-acid-responsive seizure; GABA, gamma aminobutyric acid; IV, intravenous; MTHF, 5-methyl tetrahydrofolate; P2C, piperideine-2-carboxilic acid; P6C, piperideine-6-carboxylate; PDE, pyridoxine-dependent epilepsy; PIP, pipecolic acid; PLP, pyridoxal 5' phosphate; PNPO, pyridoxine 5' phosphate oxidase enzyme

KEYWORDS epilepsy; inborn errors of metabolism; neonatal; pediatric

J Pediatr Pharmacol Ther 2019;24(5):398-405

DOI: 10.5863/1551-6776-24.5.398

\section{Introduction}

Neonates remain at high risk for seizures with an incidence as high as 1 in 1000 live births due to a variety of factors including hypoxic-ischemia, stroke, and intracranial hemorrhage.,2 Neonatal seizures should be treated as emergencies and provoke rapid diagnostic evaluation. Differential diagnoses include structural, metabolic, and genetic causes. There are diverse errors in metabolism, but those of particular interest are related to a deficiency in vitamins or cofactors including pyridoxal phosphate, pyridoxine, folinic acid, or biotin. The focus of this review will be to discuss the pharmacotherapy, including the most updated published efficacy and safety data, involved in treating refractory epilepsy as a result of metabolic errors. A literature search was performed by using MEDLINE (1962-April 2018). Combinations of the following search terms were used: pediatric epilepsy inborn metabolic errors, pyridoxine, folinic acid, biotin. References from retrieved articles were manually searched for additional citations. Clinicaltrials.gov was searched for ongoing research. All English-language studies and reviews assessing the efficacy and/or safety were included and evaluated. Randomized controlled trials, observational studies, review articles, and case reports involving pediatric patients with epilepsy who were treated with folinic acid, biotin, pyridoxine, or pyridoxal 5' phosphate were included in the review. No studies were excluded as this was intended as a literature review versus a systematic review.

\section{Pyridoxine-Dependent Epilepsy}

Pathophysiology. Initially noted by Hunt et $\mathrm{al}^{3}$ in 1954, pyridoxine-dependent epilepsy (PDE) is a rare autosomal recessive cause of metabolic epilepsy typically seen in neonates. The incidence of this condition is around 1:300,000 to 1:750,000, and it can affect multiple siblings. ${ }^{4-6}$ This disease is characterized by an error in lysine catabolism as a result of a deficiency in alpha-aminoadipic semialdehyde (AASA) dehydrogenase otherwise known as antiquitin (Figure). ${ }^{2,7}$ Piperideine-6-carboxylate, which inactivates pyridoxal phosphate, subsequently accumulates. Pyridoxal phosphate is the activated form of pyridoxine and a cofactor for numerous reactions and enzymes. Glutamic acid decarboxylase is dependent upon pyridoxal phosphate as a cofactor and is involved in the synthesis of gamma aminobutyric acid (GABA), which plays a role in the hyperexcitability of neurons. This is the assumed mechanism by which pyridoxine treats seizures.

Clinical Presentation. Most patients present with this condition shortly after birth, though atypical presentations may include seizures in late infancy until 3 years of age. ${ }^{8}$ Seizures may also occur in utero. ${ }^{6}$ Intravenous pyridoxine has a quick onset of action, though studies have shown that patients who appear to respond to IV pyridoxine by cessation of seizures after administration do not always have true PDE.? Often, it is given empirically with unknown etiology of neonatal seizures. Definitive diagnosis is through genetic testing. Recurrence may occur when pyridoxine treatment is withdrawn, 
Figure. Lysine catabolism.

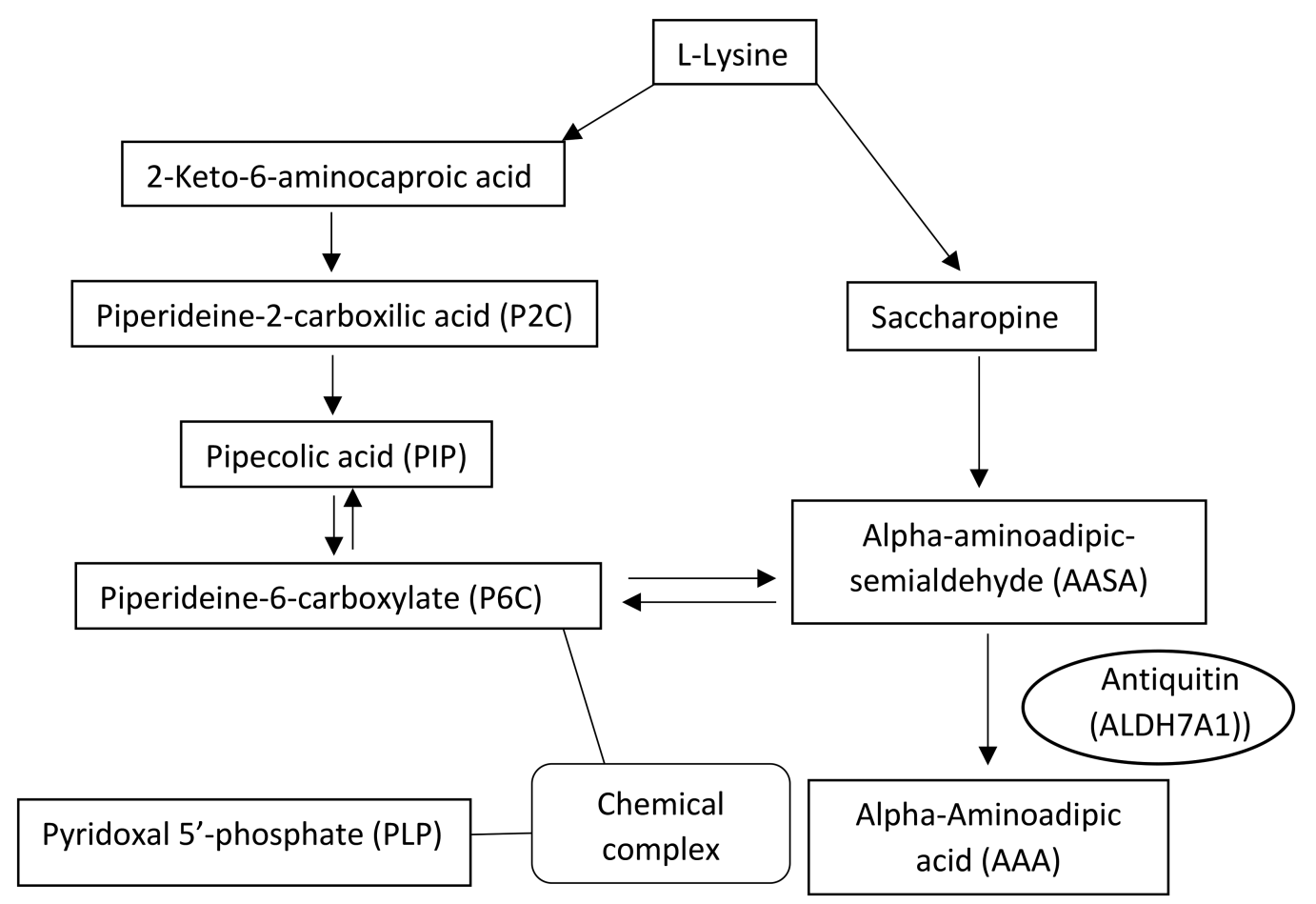

ALDH7A1, aldehyde dehydrogenase

though some atypical presentations include a lack of responsiveness or requirement of the use of additional antiepileptics. ${ }^{8}$ Patients' multiple seizure types and vague symptoms including tremors, abdominal distension, emesis, irritability, sleeplessness, hyperalertness, and abnormal eye movements present challenges in diagnosis., ${ }^{6,7}$ In some patients the condition may present as looking like hypoxic ischemic injury, a frequent cause of neonatal seizures. ${ }^{8}$

Long-term Outcomes. Pyridoxine-dependent seizures are associated with adverse neurologic outcomes ranging from mild to severe, affecting language as well as motor performance..$^{6,9-12}$ Basura et $a^{10}$ described an array of neurodevelopmental handicaps. Severe problems were more frequently seen in those individuals who had seizures despite pyridoxine maintenance treatment. There was no association between neurodevelopmental outcomes and seizure type, age of seizure onset, and delay time until PDE was diagnosed. Rankin et $\mathrm{al}^{6}$ described 3 children with PDE and moderate to severe learning disabilities. ${ }^{6}$ Bok et $\mathrm{al}^{13}$ in a retrospective cohort reported compromised cognitive development in the majority of the cohort of 14 patients $(n=10)$. Seizures were well controlled with pyridoxine monotherapy, suggesting that appropriate seizure control does not decrease the risk of adverse neurodevelopmental outcomes. There was no correla- tion between long-term neurodevelopmental outcomes and age that treatment was initiated.

Treatment. Pyridoxine supplementation is the treatment of choice. Evidence for its use in PDE is mixed but confounded by the fact that genetic testing results are often not reported and patients are on multiple antiseizure medications. Cirillo ${ }^{7}$ reported on a case of true PDE diagnosed from genetic testing. This patient born at 38 weeks was initiated on oral pyridoxine on the fifth day of life, which was titrated up to $150 \mathrm{mg}$ once daily while she was being weaned off concomitant phenobarbital. No information was available regarding patient weight. The patient did not have any further seizures and at follow-up had normal neurological development, suggesting efficacy in the case of true PDE.

Lin et $\mathrm{al}^{14}$ described the case of a 1-month-old female with unreported weight and recurrent and persistent seizures despite treatment with phenobarbital. Oral pyridoxine $50 \mathrm{mg} /$ day was initiated and titrated up to 200 mg/day; phenobarbital was tapered off. Initiating with oral pyridoxine is unusual; typically, IV pyridoxine would be initiated first and then transitioned to the oral form, so this strategy is unusual. This also supports the idea that pyridoxine may not only treat seizures but also allow for weaning of concomitant medications. Garty et $\mathrm{al}^{5}$ described a case of a $2.8 \mathrm{~kg}$ term female neonate, otherwise healthy, who developed generalized 
Table. Pharmacotherapy Considerations for Vitamin-Dependent Epilepsies

\begin{tabular}{|c|c|c|c|}
\hline Deficiency & Recommended Initial Doses & Dosage Forms/Routes Available & Estimated Cost (\$)* \\
\hline Pyridoxine & $50 \mathrm{mg} /$ day or $15-30 \mathrm{mg} / \mathrm{kg} /$ day & $\begin{array}{l}\text { Intravenous solution } \\
\text { Oral capsules } \\
\text { Oral tablets }\end{array}$ & $5-10$ \\
\hline Pyridoxal 5' phosphate & $30 \mathrm{mg} / \mathrm{kg} / \mathrm{day}$ & Oral capsules & $10-20$ \\
\hline Biotin & $5-10 \mathrm{mg}$ & $\begin{array}{l}\text { Oral capsules }^{\dagger} \\
\text { Oral tablets } \\
\text { Oral suspension } \\
\text { Oral solution }\end{array}$ & $5-10$ \\
\hline Folinic acid & $2.5-5 \mathrm{mg}$ twice daily & $\begin{array}{l}\text { Intravenous solution } \\
\text { Oral tablet } \\
\text { Oral compounded suspension }\end{array}$ & 280 \\
\hline
\end{tabular}

* Estimated cost in US dollars based on 100 oral solid dosage forms.

${ }^{+}$Recommended dosage forms.

convulsions 6 hours after birth and was unresponsive to barbiturates and phenytoin. This patient responded to 5-mg intramuscular pyridoxine, and oral pyridoxine $7.5 \mathrm{mg}$ daily was then initiated. Doses of pyridoxine were titrated down and seizures recurred at 1-mg oral daily dosing. This was the only study found that used intramuscular dosing, with no conversion data between the 2 dosage forms, thus it is not the recommended method based on body of evidence. Seizures recurring at lower doses suggest that pyridoxine was efficacious treatment, even without genetic results.

There is a wide range of dosing for pyridoxine. It is recommended to initiate dosing at 15 to $30 \mathrm{mg} / \mathrm{kg} / \mathrm{day}$, though dosing may be as low as $1 \mathrm{mg} / \mathrm{kg} / \mathrm{day} .{ }^{6}$ Basura et $\mathrm{al}^{10}$ noted that an average maintenance daily dose of pyridoxine was $354 \mathrm{mg}$ or $13.1 \mathrm{mg} / \mathrm{kg}(\mathrm{N}=70)$. Expert opinion cites dosing up to 500-mg IV pyridoxine in a single dose. ${ }^{15}$ Seizures respond fairly quickly to treatment, even within minutes of the IV dose.

Monitoring. Patients should be monitored for apneas following the first dose of pyridoxine regardless of formulation and be monitored via continuous electroencephalography while following cardiorespiratory status. ${ }^{5,6}$ With higher daily doses, specifically those higher than $200 \mathrm{mg}$, patients may also experience sensory neuropathies. ${ }^{2,6}$ While not all studies document changes in electroencephalography, some data suggest increases in intelligence quotient particularly with higher doses of pyridoxine treatment up to $18 \mathrm{mg} /$ $\mathrm{kg} /$ day. ${ }^{9}$ Practitioners should be aware that doubling the dose during acute illness to prevent seizure onset is appropriate. ${ }^{15}$ Additionally, if taking other anticonvulsants such as phenytoin or fosphenytoin, further monitoring may be required owing to drug interactions and compromised effectiveness of the former. Duration of therapy, given the genetic basis for seizures, would be lifelong.

Availability and Administration. Pyridoxine is available as both an IV solution $100 \mathrm{mg} / \mathrm{mL}$ and in oral form as a capsule or tablet in 25, 50, 100, 200 (extended- release), and $250 \mathrm{mg}$. Pricing for the capsules and tablets are anywhere from $\$ 5$ to $\$ 10$ for a quantity of 100 , and this product is available over the counter.

\section{Pyridoxine 5' Phosphate Oxidase Deficiency-}

Pathophysiology. In pyridoxine 5' phosphate oxidase deficiency, there is a homozygous or heterozygous mutation on chromosome $17 q 21$, resulting in a nonfunctional pyridoxine 5' phosphate oxidase enzyme (PNPO). PNPO converts phosphorylated pyridoxine and pyridoxamine phosphate to pyridoxal 5' phosphate or PLP. ${ }^{8}$ PLP is a cofactor for many enzymes including glutamic acid decarboxylase (Figure). When concentrations are low, normal GABA synthesis is interrupted and hyperexcitability of neurons and seizures can occur. PNPO represents an error in the typical synthesis of pyridoxal phosphate, rather than enhanced inactivation. PLP is also referred to as activated pyridoxine, based on its function as a cofactor and the conversion of pyridoxine to the active, usable form in reactions. Diagnosis is typically confirmed with mutation analysis of the PNPO gene. ${ }^{2}$

Clinical Presentation. Patients present somewhat differently than those with pyridoxine-related seizures. ${ }^{15}$ These patients are often born prematurely with seizures occurring more frequently in utero versus patients with pyridoxine-related seizures. During the postnatal period, neonates will often experience hypoglycemia, lactic acidosis, seizures, and encephalopathy.

Treatment. Treatment for PNPO is pyridoxal phosphate supplementation (PLP). Pyridoxal 5' phosphate is the activated form of pyridoxine and eliminates the activation step by the mutated non-functional PNPO enzyme. ${ }^{16}$ There are 2 case reports, a case series, and 1 open label prospective study evaluating PLP in children with a range of dosing. Kuo and Wang ${ }^{17}$ described a case of a preterm female infant (gestational age 35 weeks) in Taiwan weighing $1795 \mathrm{~g}$ who developed seizures at hour 3 of life that were controlled with 40 - 
mg IV PLP once followed by $10 \mathrm{mg}$ IV every 6 hours. Seizures recurred when switching to oral pyridoxine. Repeated dosing of 50-mg IV PLP stopped seizures, which were subsequently controlled with $50 \mathrm{mg} \mathrm{IV}$ every 6 hours of PLP or 30 mg/kg/day. Pyridoxal 5' phosphate was converted to oral form and concurrent antiseizure medications were discontinued without breakthrough seizures. This study suggests that this was a PNPO deficiency as opposed to PDE. Use of this medication was also beneficial because other antiseizure medications could be discontinued while controlling seizures. Clayton et $\mathrm{al}^{18}$ described a male infant at 35 weeks' gestation who developed seizures on day of life 1 with no response to anticonvulsants or oral pyridoxine $100 \mathrm{mg}$ twice daily for 5 days. Seizures stopped when 50-mg PLP via nasogastric tube was initiated, and this empiric treatment was effective and similar in dosing to the previous studies for loading dose. He was maintained on $30 \mathrm{mg} / \mathrm{kg} / \mathrm{day}$ PLP-and vigabatrin, with only infrequent seizures. The authors did not provide information on how the dose was divided or follow-up data, beyond noting microcephaly and developmental delay. While genetic information was unavailable, response to initial treatment suggests efficacy though the causes of seizures in some affected patients may be multifactorial and thus require multiple medications.

In an open prospective study in Taiwan, Wang et al ${ }^{19}$ evaluated the efficacy of PLP therapy in children with intractable epilepsy. Inclusion criteria were seizures more than once daily and epilepsy for more than 6 months despite taking at least 3 classes of antiepileptic medications. Patients $(\mathrm{N}=94)$ were given IV PLP 10 $\mathrm{mg} / \mathrm{kg} /$ day once followed by $10 \mathrm{mg} / \mathrm{kg} /$ day in 4 divided doses over 3 days. If seizures recurred, another 40 $\mathrm{mg} / \mathrm{kg}$ IV bolus was administered and daily dosing increased to $50 \mathrm{mg} / \mathrm{kg} /$ day. If seizures did not occur at the end of 3 days, patients were changed over to oral pyridoxine. Patients were thus evaluated for more of a PDE diagnosis as opposed to strictly a PNPO deficiency. If seizures recurred on oral pyridoxine, suggesting PNPO deficiency, patients were reloaded with IV PLP and changed to oral PLP once the condition was controlled. The same principle of treating empirically without a genetic diagnosis, as done in previously mentioned studies, was used here. Antiseizure drugs were then tapered when patients were free of seizures for 1 month. Eleven patients (11.7\%) responded to IV PLP, with 8 of the $11(72 \%)$ requiring the increased dose of $50 \mathrm{mg} / \mathrm{kg} /$ day. All 11 responders were started on oral pyridoxine, but 6 of the 11 patients (54.5\%) experienced seizure recurrence. Those 6 patients who failed therapy were then initiated on oral PLP. Fifty percent of patients on oral PLP maintenance therapy and $40 \%$ of patients on oral pyridoxine remained seizure-free after other antiepileptic drugs were tapered off. Few patients responded to IV PLP but of those who did, most required higher doses and about half required PLP as maintenance therapy. Once transitioned to a stable oral regimen of pyridoxine or PLP, patients remained seizure-free about $50 \%$ of the time without other antiepileptic drugs, which is a standard barometer of antiseizure medication success. The average final dosage of oral PLP was $30 \mathrm{mg} / \mathrm{kg} / \mathrm{day}$, which was higher than the average dose of pyridoxine in the maintenance group of $18 \mathrm{mg} / \mathrm{kg} /$ day. There were no toxicities noted. A dose of $30 \mathrm{mg} / \mathrm{kg} /$ day of oral PLP for maintenance dosing is safe and effective for those unresponsive to pyridoxine. None of the patients were genetically tested, and the authors did not state a position on why only a few patients responded.

More recently, a case series was published on seizures related to PLP in pediatric patients. ${ }^{20}$ The authors investigated PLP concentrations within the cerebrospinal fluid (CSF), as decreased levels of PLP in the CSF have been linked with seizure development. CortesSaladelafont et $\mathrm{al}^{20}$ looked at patients $(\mathrm{N}=10)$ between 3 months and 15 years of age with low-normal levels of PLP in the CSF who received PLP 10 to $30 \mathrm{mg} / \mathrm{kg} /$ day as used in the reports by Clayton et $\mathrm{al}^{18}$ and Wang et al. ${ }^{19}$ Patients initially experienced symptoms at 17 hours of life to 6 years of age. Only 1 patient had a mutation in the PNPO gene, and all patients were on concurrent antiepileptic medications. One non-responder patient was diagnosed with Phelan-McDermid syndrome, with a predisposition to seizures, and would unlikely require PLP monotherapy for seizure control. Four of the 10 patients had subjective or objective improvement, 4 had no improvement, and 2 experienced adverse effects, namely, gastrointestinal distress. This improvement was seen gradually over the course of a few days to weeks. None of the patients continued with PLP supplementation beyond 3 months. This most recent study fails to demonstrate successful use of PLP. However, only 1 of the 10 patients had a true PNPO deficiency, which may explain the lack of effectiveness.

Adverse Events and Monitoring. Adverse events documented in limited studies include gastrointestinal distress and elevation in liver enzymes. ${ }^{20}$ These were reversible upon discontinuation of the agent. It is recommended to monitor liver function tests in these patients, in addition to self-reported seizures.

Availability and Administration. There are some reservations in the use of PLP. Intravenous PLP is not available in the United States. Oral PLP is available, but as an unregulated dietary supplement available over the counter, there are concerns with the purity and consistency of the product. Furthermore, as a dietary supplement, it is unlikely to be on hospital formularies. If a patient were to be admitted to the hospital, this medication may need to be supplied as a non-formulary product. Oral PLP costs are about double those of pyridoxine products. Notably, several patients in the study of Wang et al19 had to reduce oral maintenance 
doses owing to cost, so the financial barrier may compromise therapeutic efficacy. Liver function tests should be monitored. Adverse events are largely related to mild gastrointestinal disturbances. ${ }^{20}$ These may also potentially be avoided by keeping the starting dose low at $10 \mathrm{mg} / \mathrm{kg} / \mathrm{day}$ and titrating as tolerated.

\section{Biotinidase Deficiency}

Pathophysiology. Biotin (vitamin B7, formerly known as Vitamin $\mathrm{H}$ ) is a necessary vitamin in the production of strong nails and hair but also in the metabolism of amino acids, fats, and glucose. Biotin is found in a protein-bound form in most foods. Biotinidase deficiency is an autosomal recessive disorder with an estimated incidence of 1 in 60,000 newborns that is easily treated with oral biotin. ${ }^{21}$ The metabolism process involves the conversion of the free protein-bound biotin into biocytin by proteases and peptidases. Biocytin is then converted back to free biotin by an enzyme biotinidase. ${ }^{22}$ Profound biotinidase deficiency is defined as less than $10 \%$ of normal activity, while partial biotinidase deficiency is between 10\% and 30\% of normal enzyme activity.

Clinical Presentation. Most patients are identified with this deficiency at birth. In 1984 an assay, developed to assess biotidinase activity, was piloted in Virginia's newborn screening program. Of 81,243 newborn infants screened, 4 children were identified with a biotinidase deficiency. ${ }^{23,24}$ While most deficiencies are now diagnosed on newborn screening, for untreated patients, a biotin deficiency will typically present in the first few months of life, ranging in symptoms from skin rash and alopecia to seizures and hypotonia. ${ }^{22,25}$ In some cases, untreated older children have presented with ocular deficits and motor limb weaknesses. ${ }^{25}$ In those with a profound biotinidase deficiency, seizures of all types can be seen in upwards of $70 \%$ of patients. The proposed mechanism is thought to be due to irregular myelination. ${ }^{26}$ While seizures are generally tonic-clonic in nature (56\%), others have reported myoclonic, infantile spasms and Ohtahara syndrome. ${ }^{27}$ Lactic acidosis, hyperventilation, and laryngeal stridor are seen in severe cases. Ultimately, low biotinidase activity will confirm the diagnosis.

Treatment. Prior to widespread newborn screening, Salbert et al ${ }^{28}$ reviewed 78 children to characterize seizures associated with biotinidase deficiency. More than half had a history of seizures (55\%) with half of those patients having seizures uncontrolled with conventional antiepileptic agents. Sixteen patients with continued seizures were treated with oral biotin therapy at doses of 5 to $10 \mathrm{mg}$. Seventy-five percent of those patients had cessation of seizures within 24 hours. The authors concluded that a trial of biotin should be considered in any infant with unexplained seizures.

Most manifestations are easily treated by replacement of biotin; seizures should quickly cease within hours to a single day and any cutaneous manifestations will improve over the course of several weeks to months. ${ }^{26}$ For patients in which long-standing biotin deficiency has resulted in vision loss, permanent damage may be resistant to treatment. Grunewald et $a^{29}$ reviewed imaging abnormalities seen in 5 untreated biotidinase-deficient patients. In all patients, delayed myelination was common along with white matter abnormalities potentially associated with ischemia due to metabolic insult. Even with treatment, 3 of the 5 patients had residual complications including blindness, seizures, hearing impairment, and developmental delays. In another case report, Mico and colleagues ${ }^{27}$ reported a 2.5 -month-old patient who presented with abnormal movements, failure to thrive, and neurologic depression. ${ }^{27}$ She was found to have a severe biotinidase deficiency and was initially treated with biotin 20 mg daily. The seizures quickly abated but her microcephaly and cognitive impairment continued. Despite biotin treatment and normal biotin plasma levels during her childhood, she had recurrent febrile and afebrile seizures treated with phenobarbital and levetiracetam. This case demonstrates that although seizures may initially stop once the biotin is replaced, patients may develop a secondary epileptic condition along with other neurologic sequelae. The authors in both reports stressed the importance of early diagnosis and treatment with biotin replacement.

The universal treatment for patients with this genetic condition is replacement using oral biotin. For those with profound deficiency, regardless of weight or age, widely recommended dosing for biotin is 5 to $10 \mathrm{mg}$ orally daily. ${ }^{24,26}$ With no adverse reactions associated with the use of biotin, there has not historically been a weight-based dose or distinct dose recommendation for infants. For patients with partial deficiency, it is less clear which patients may require treatment. Owing to the favorable safety profile, most partial-deficiency patients will also be treated with supplemental biotin doses similar to those for profound deficiency. ${ }^{30}$ Higher dosages of 15 to $20 \mathrm{mg}$ per day may be necessary in some patients especially those with hair loss and developmental delays.

Availability and Administration. Biotin tablets are readily available at local pharmacies, may be found on hospital formularies, and are relatively inexpensive. Tablets, which can be crushed, and capsules are recommended for all ages to ensure consistent dosing. Solutions and suspensions are noted to settle upon refrigeration. Egg whites will bind and reduce the bioavailability of biotin. ${ }^{26}$ Concomitant valproic acid therapy may also impair liver function, thus decreasing biotinidase activity. It is recommended not to use valproic acid in biotinidase deficiency patients. ${ }^{31}$ Therapy is lifelong and patients who have stopped taking their medication will have a recurrence of symptoms within weeks. ${ }^{26}$ 


\section{Folinic Acid Deficiencies}

Pathophysiology. Cerebral folate deficiencies are a cluster of neurological conditions resulting from a deficiency in 5-methyl tetrahydrofolate (MTHF) in the central nervous system yet with normal metabolism in the periphery. A folinic-acid-responsive seizure (FARS) is classified as a cerebral folate deficiency that is responsive to replacement of folic acid derivatives. FARS has been associated with both genetic and autoimmune mechanisms. ${ }^{26,32,33}$ In 1995, researchers identified this new class of seizure that responded to folinic acid. ${ }^{34}$ Since that time, many reports have corroborated this finding. 33,35,36 Two distinct disease mechanisms of FARS have been proposed: autoimmune folate antibodies and mutation of the FOLR1 gene. In the autoimmune process, autoantibodies to folic acid develop, while with FOLR1 gene mutation, the transport of folate to the central nervous system is disrupted, resulting in altered neuronal metabolism. ${ }^{26}$ Over time, neurons progressively lose structure and function.

More recently, FARSs have been linked to pyridoxinedependent seizures. Gallagher and colleagues ${ }^{37}$ analyzed CSF samples of patients with presumed FARS and concluded that these are identical to the pathophysiology of PDEs, an alpha-AASA dehydrogenase deficiency associated with mutations in the ALDH7A1 gene. This information suggests that treatment with both pyridoxine and folinic acid for patients with this deficiency may be better suited to prevent long-term sequelae.

Clinical Presentation. Patients with autoimmune pathophysiology present with clinical symptoms such as irritability and sleep disturbances around the age of 4 months. Progressive symptoms will include seizures, dyskinesia, and psychomotor retardation. Seizures will present in a variety of forms including myoclonic, absence, and generalized tonic-clonic seizures. Patients with FOLR1 gene mutation will typically present later than patients with autoantibodies, typically after infancy. The presentation is similar, however with seizures and other neurological expressions, developmental delay, and hypotonia. ${ }^{26}$

Treatment. Regardless of pathophysiology, FARS will typically respond to folinic acid doses of 0.5 to $1 \mathrm{mg} / \mathrm{kg} /$ day. The earliest reported case was in a 6-month-old female who had onset of status epilepticus at 5 days of life. ${ }^{34}$ The seizures were only being partially controlled with conventional anticonvulsants including pyridoxine, phenobarbital, and valproic acid. Oral folinic acid was initiated at 6 months of life at a dose of $2.5 \mathrm{mg}$ twice daily. Within 24 hours, there was not only cessation of seizure activity but also a decrease of $75 \%$ in the concentration of an unknown compound in the CSF. By age 4 years, the patient was taking $15 \mathrm{mg}$ of folinic acid orally twice daily along with other anticonvulsants.

Several years later, the same author ${ }^{35}$ presented cases of 2 more infants whose neonatal seizures responded to folinic acid. The first was a 6-month-old female who had a history of seizures since birth, initially controlled by phenobarbital. She presented in status epilepticus with no response to conventional medications or pyridoxine. The authors started folinic acid at $5 \mathrm{mg}$ orally twice a day and seizures abated within 24 hours. By age 3 years, the patient remained on folinic acid at a dose of 20 mg twice daily with other anticonvulsants. The second patient was similar but his therapy began at 9 weeks of age. Folinic acid at a dose of 2.5 mg orally twice daily was initiated. Although slower to respond, the patient became seizure-free at 5 days following therapy. Although requiring increases in dosage up to $10 \mathrm{mg}$ twice daily, at 15 months of age he was reported to be seizure-free, off other anticonvulsants, and with only mild diffuse hypotonia. ${ }^{35}$

In 2003 , Frye and colleagues ${ }^{36}$ reported a single case in a 3-month-old infant. This infant was initially treated with phenobarbital and fosphenytoin in the neonatal intensive care unit, with good control. The patient was discharged on monotherapy with phenobarbital. When weaned in the outpatient setting, the patient experienced a generalized seizure. At this time, the patient was reloaded with phenobarbital but continued to have intermittent seizures over the next 2 weeks. Vitamin therapies including thiamine, riboflavin, pyridoxine, and cyanocobalamin were initiated with initial benefit but with continued lip smacking, tonic-clonic activity of the extremities, and leftward eye deviation. Cerebrospinal fluid collected during a previous admission was positive for similar unknown compounds previously found to be diagnostic for FARSs. Folinic acid was started at a dose of $4 \mathrm{mg} / \mathrm{kg} /$ dose divided twice daily. Seizures abated within 24 hours. Unfortunately, weeks later, seizures resumed and the patient ultimately succumbed to the illness. The authors suggest that when folinic acid deficiencies are suspected, a complete workup including CSF amino acids, ammonia, and neurotransmitters should be completed to allow for initiation of the correct therapies early in the disease course.

In a larger study, Ramaekers and Blau ${ }^{33}$ treated 20 patients with low MTHF levels in the CSF All were given folinic acid at an oral dose of 0.5 to $1 \mathrm{mg} / \mathrm{kg}$ daily. Patients with no clinical effect during the first 4 to 6 months had a lumbar puncture to reassess MTHF levels. Dose adjustments were made, up to 2 to $3 \mathrm{mg} /$ $\mathrm{kg} /$ day. In a subsequent publication, the authors note that patients with FOLR1 mutations may require higher daily doses, up to 3 to $5 \mathrm{mg} / \mathrm{kg} /$ day, to correct MTHF CSF levels. ${ }^{38}$ The researchers noted that the group of children diagnosed and treated before 6 years of age had a more favorable neurologic recovery along with seizure cessation. Those older than 6 years had a delayed and incomplete response. The authors concluded, however, that treatment did halt the progression of any further decline in neurologic status.

Availability and Administration. Folinic acid is avail- 
able in both IV and oral dosage forms. From the case reports above, dosing is typically initiated at 2.5 to 5 $\mathrm{mg}$ orally twice daily but will be titrated up on the basis of response. If patients are unable to tolerate administration with a tablet, an extemporaneous solution may be compounded. ${ }^{39}$ The cost of treatment tends to be more expensive than that of other vitamin therapies but as with others, compliance is necessary. Folic acid should not be substituted for folinic acid, as it can paradoxically worsen the concentration of reduced folate derivatives in the central nervous system. ${ }^{34}$ Although considered to be generally safe at the doses given for this indication, gastrointestinal upset and mental status changes have been associated with the medication. Some patients report hyperactivity, while others have sleep disturbances and trouble concentrating.

\section{Discussion}

Literature on treatment of inborn errors of metabolism and refractory epilepsy are composed of largely case series, but dosing is relatively consistent across studies. Other considerations include dosage form limitations, adverse events, and therapeutic monitoring for seizures and the medications used. Over-thecounter preparations that are not regulated by the US Food and Drug Administration may have differences in quality. Products with a United States Pharmacopeia seal have been verified for quality and should be used preferentially. Consultation with a pharmacist is helpful to determine the best product and the availability for oral liquid compounds.

It is reasonable to consider pyridoxine as well as folinic acid as initial therapies for patients with refractory epilepsy as a neonate. If a patient fails to respond but inborn errors related to pyridoxine are suspected, then oral PLP 30 to $50 \mathrm{mg} / \mathrm{kg} /$ day may be used. Many of these therapies are lifelong.

Neurological sequelae may remain for many of these patients, even with controlled seizures, so while these therapies may be helpful for seizure control, longitudinal outcomes must still be cautiously interpreted. While genetic testing is recommended for all patients, the results of this testing may be delayed. Earlier initiation of these therapies, given their low adverse event profile, is recommended, to be administered initially for refractory seizures with the potential to optimize patient outcomes. The body of literature supporting the use of agents like pyridoxine or folinic acid also suggests that most of the time, this initial empiric treatment is being used. Patients also may have a multifactorial etiology to seizures, so while these agents may be useful to control one aspect of seizures, additional therapies may be required.

\section{Conclusion}

Seizures in the neonatal population stem from a variety of causes, including inborn errors of metabolism. Many of these patients may initially be clinically identified by symptoms of refractory epilepsy, with follow-up through genetic testing. Four of the more common inborn errors, namely, those affecting folinic acid, biotin, pyridoxine, and pyridoxal 5' phosphate, were included in this review. Though future studies would be beneficial to optimize what we understand about dosing and efficacy, current literature supports the use of these agents with appropriate monitoring in order to optimize long-term outcomes and seizure control.

\section{ARTICLE INFORMATION}

Affiliations Department of Pharmacy (ASC), Bellevue Hospital, New York, NY, Department of Pharmacy (CTC), University of Florida Health-Shands, Gainesville, FL, Department of Pharmacotherapy and Translational Research (CTC), University of Florida College of Pharmacy, Gainesville, FL

Correspondence Anna S. Cosnahan, PharmD; annacosnahan2@gmail.com

Disclosure The authors declare no conflicts or financial interest in any product or service mentioned in the manuscript, including grants, equipment, medications, employment, gifts, and honoraria.

Accepted March 8, 2019

Copyright Published by the Pediatric Pharmacy Advocacy Group. All rights reserved.

For permissions, email: matthew.helms@ppag.org

\section{REFERENCES}

1. Glass HC. Neonatal seizures: advances in mechanisms and management. Clin Perinatol. 2014;41(1):177-190.

2. Rahman S, Footitt EJ, Varadkar S, et al. Inborn errors of metabolism causing epilepsy. Dev Med Child Neurol. 2013;55(1):23-36.

3. Hunt AD, Stokes J Jr, McCrory WW, et al. Pyridoxine dependency: a report of a case of intractable convulsions in an infant controlled by pyridoxine. Pediatrics. 1954;13(2):140-145.

4. Gospe SM Jr. Pyridoxine-dependent seizures: new genetic and biochemical clues to help with diagnosis and treatment. Curr Opin Neurol. 2006;19(2):148-153.

5. Garty R, Yonis Z, Braham J, Steinitz K. Pyridoxinedependent convulsions in an infant. Arch Dis Child. 1962;37(191):21-24.

6. Rankin PM, Harrison S, Chong WK, et al. Pyridoxinedependent seizures: a family phenotype that leads to severe cognitive deficits, regardless of treatment regime. Dev Med Child Neurol. 2007;49(4):300-305.

7. Cirillo M. Case report: intravenous and oral pyridoxine trial for diagnosis of pyridoxine-dependent epilepsy. Pediatrics. 2015;136(1):e257-e261. 
8. Gospe SM. Pyridoxine-dependent epilepsy. In: Adam MP, Ardinger $\mathrm{HH}$, Pagon RA, et al, eds. Gene Reviews [Internet]. Seattle, WA: University of Washington; 2001.

9. Baxter P. Pyridoxine dependent and pyridoxine responsive seizures. In: Baxter P, ed. Vitamin Responsive Conditions in Pediatric Neurology. London: MacKeith Press; 2001:109-165.

10. Basura GJ, Hagland SP, Wiltse AM, et al. Clinical features and the management of pyridoxine-dependent and pyridoxine-responsive seizures: review of 63 North American cases submitted to a patient registry. Eur $J$ Pediatr. 2009;168(6):697-704.

11. Baxer P, Griffiths P, Kelly T, et al. Pyridoxine-dependent seizures: demographic, clinical, MRI and psychometric features, and effect of dose on intelligence quotient. Dev Med Child Neurol. 1996;38(11):998-1006.

12. Baynes K, Tomaszewski FS, Gospe SM Jr. Pyridoxinedependent seizures and cognition in adulthood. Dev Med Child Neurol. 2003;45(11):782-785.

13. Bok LA, Halbertsma FJ, Houterman S, et al. Long-term outcome in pyridoxine-dependent epilepsy. Dev Med Child Neurol. 2012;54(9):849-854.

14. Lin J, Lin K, Rodrigues M, et al. Pyridoxine-dependent epilepsy initially responsive to phenobarbital. Arq Neuropsiquiatr. 2007;65(4):1026-1029.

15. Gospe SM. Neonatal vitamin-responsive epileptic encephalopathies. Chang Gung Med J. 2010;33(1):1-12.

16. Vrolijk MF, Opperhuizen A, Jansen EH, et al. The vitamin B6 paradox: supplementation with high concentrations of pyridoxine leads to decreased vitamin B6 function. Toxicol In Vitro. 2017;44:206-212.

17. Kuo MF, Wang HS. Pyridoxal phosphate-responsive epilepsy with resistance to pyridoxine. Pediatr Neurol. 2002;26(2):146-147.

18. Clayton PT, Surtees RAH, DeVile C, et al. Neonatal epileptic encephalopathy. Lancet. 2003;361(9369):1614. doi:10.1016/s0140-6736(03)13312-0.

19. Wang HS, Kuo MF, Chou ML, et al. Pyridoxal phosphate is better than pyridoxine for controlling idiopathic intractable epilepsy. Arch Dis Child. 2005;90(5):512-515.

20. Cortes-Saladelafont E, Molero-Luis M, HSJD Working Group, et al. Pyridoxal phosphate supplementation in neuropediatric disorders. Semin Pediatr Neurol. 2016;23(4):351-358.

21. Wolf $\mathrm{B}$. Worldwide survey of neonatal screening for biotinidase deficiency. J Inherit Metab Dis. 1991;14(6):923-927.

22. Ods.od.nih.gov. 2018. Office of Dietary Supplements Biotin [online]. https://ods.od.nih.gov/factsheets/BiotinHealthProfessional. Accessed June 23, 2019.

23. Strovel ET, Cowan TM, Scott Al, et al. Laboratory diagnosis of biotinidase deficiency, 2017 update: a technical standard and guidelines of the American College of Medical Genetics and Genomics. Genet Med. 2017.19(10):1-10.
24. Wolf B, Heard GS, Jefferson LF, et al. Clinical findings in four children with biotinidase deficiency detected through a statewide neonatal screening program. NEngl J Med. 1985;313(1):16-19.

25. Cowan TM, Blitzer MG, Wolf B. Technical standards and guidelines for the diagnosis of biotinidase deficiency. Genet Med. 2010;12(7):464-470.

26. Agadi S, Quach MM, Haneef Z. Vitamin-responsive epileptic encephalopathies in children. Epilepsy Res Treat. 2013;2013:510529. doi:10.1155/2013/510529.

27. Mico SI, Jimenez RD, Salcedo EM, et al. Epilepsy in biotinidase deficiency after biotin treatment. JIMD Rep. 2012;4:75-78.

28. Salbert BA, Pellock JM, Wolf B. Characterization of seizures associated with biotinidase deficiency. Neurology. 1993;42(7):1351-1355.

29. Grunewald S, Champion MP, Leonard JV, et al. Biotinidase deficiency: a treatable leukoencephalopathy. Neuropediatrics. 2004;35(4):211-216.

30. Wolf B. Biotinidase deficiency. In: Adam MP, Ardinger HH, Pagon RA, et al, eds. GeneReviews ${ }^{\circledR}$ [Internet]. Seattle, WA: University of Washington, Seattle; 2000:1993-2018. https://www.ncbi.nlm.nih.gov/books/NBK1322/. Updated June 9, 2016. Accessed June 23, 2019.

31. Schulpis KH, Karikas GA, Tjamouranis I, et al. Low serum biotinidase activity in children with valproic acid monotherapy. Epilepsia. 2001;42(10):1359-1362.

32. Al-Baradie RS, Chudary MW. Diagnosis and management of cerebral folate deficiency. Neurosciences (Riyadh). 2014;19(4):312-316.

33. Ramaekers VT, Blau N. Cerebral folate deficiency. Dev Med Child Neurol. 2004;46(12):843-851.

34. Hyland K, Buist NR, Powell BR, et al. Folinic acid responsive seizures: a new syndrome? J Inherit Metab Dis. 1995;18(2):177-181.

35. Torres OA, Miller VS, Buist NM, et al. Folinic acid responsive neonatal seizures. J Child Neurol. 1999;14(8):529532.

36. Frye RE, Donner E, Golja A, et al. Folinic acid-responsive seizures presenting as breakthrough seizures in a 3-month-old boy. J Child Neurol. 2003;18(8):562-569.

37. Gallagher RC, Van Hove JL, Scharer G, et al. Folinic acid-responsive seizures are identical to pyridoxinedependent epilepsy. Ann Neurol. 2009;65(5):550-556.

38. Ramaekers V, Sequeira JM, Quadros EV. Clinical recognition and aspects of the cerebral folate deficiency syndromes. Clin Chem Lab Med. 2013;51(3):497-511.

39. Lam MS. Extemporaneous compounding of oral liquid dosage formulations and alternative drug delivery methods for anticancer drugs. Pharmacotherapy. 2011;31(2):164-192. 